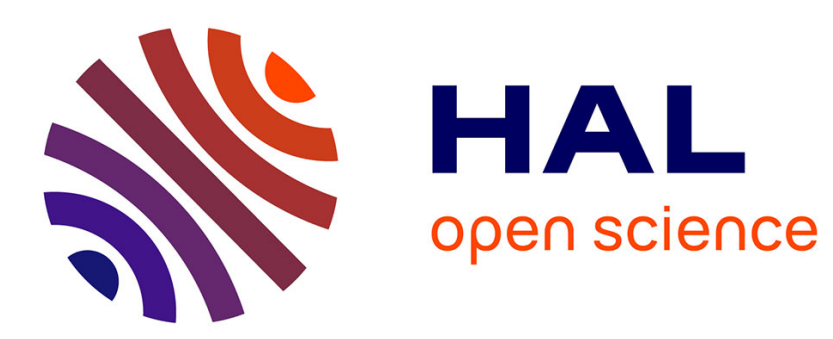

\title{
The influence of a pressure wavepacket's characteristics on its acoustic radiation
}

R. Serré, Jean-Christophe Robinet, F. Margnat

\section{To cite this version:}

R. Serré, Jean-Christophe Robinet, F. Margnat. The influence of a pressure wavepacket's characteristics on its acoustic radiation. Journal of the Acoustical Society of America, 2015, 137 (6), pp.3178-3189. 10.1121/1.4921031 . hal-02569482

\section{HAL Id: hal-02569482 \\ https://hal.science/hal-02569482}

Submitted on 11 May 2020

HAL is a multi-disciplinary open access archive for the deposit and dissemination of scientific research documents, whether they are published or not. The documents may come from teaching and research institutions in France or abroad, or from public or private research centers.
L'archive ouverte pluridisciplinaire HAL, est destinée au dépôt et à la diffusion de documents scientifiques de niveau recherche, publiés ou non, émanant des établissements d'enseignement et de recherche français ou étrangers, des laboratoires publics ou privés. 


\title{
The influence of a pressure wavepacket's characteristics on its acoustic radiation
}

\author{
R. Serré and J.-C. Robinet \\ Arts et Metiers ParisTech, DynFluid, 151 Boulevard de l'Hopital, 75013 Paris, France \\ F. Margnat ${ }^{\text {a) }}$ \\ Institut PPRIME, Department of Fluid Flow, Heat Transfer and Combustion, Université de Poitiers - \\ ENSMA - CNRS, Building B17 - 6 rue Marcel Doré, TSA 41105, 86073 Poitiers Cedex 9, France
}

\begin{abstract}
Noise generation by flows is modeled using a pressure wavepacket to excite the acoustic medium via a boundary condition of the homogeneous wave equation. The pressure wavepacket is a generic representation of the flow unsteadiness, and is characterized by a space envelope of pseudoGaussian shape and by a subsonic phase velocity. The space modulation yields energy in the supersonic range of the wavenumber spectrum, which is directly responsible for sound radiation and directivity. The influence of the envelope's shape on the noise emission is studied analytically and numerically, using an acoustic efficiency defined as the ratio of the acoustic power generated by the wavepacket to that involved in the modeled flow. The methodology is also extended to the case of acoustic propagation in a uniformly moving medium, broadening possibilities toward practical flows where organized structures play a major role, such as co-flow around cruising jet, cavity, and turbulent boundary layer flows. The results of the acoustic efficiency show significant sound pressure levels, especially for asymmetric wavepackets radiating in a moving medium.
\end{abstract}

\section{INTRODUCTION}

The goal of the present study is to estimate an acoustic efficiency of unsteady flows, defined as the rate of energy that aerodynamic fluctuations are able to leak into acoustic waves. That problem is addressed here from the acoustical point of view, that is, searching for which kind of excitation the acoustic medium responds the most (or the least) efficiently. In order to model flow induced noise, a generic excitation may be a wave whose phase speed is subsonic and whose amplitude is spatially modulated. ${ }^{1,2}$ This accounts for a localized shear region embedded in the acoustic medium, as one can find, e.g., within a spatially evolving mixinglayer: Kelvin-Helmholtz vortices, due to a harmonic forcing inflow, are convectively amplified before the pairing phenomenon occurs. The hydrodynamic pressure fluctuation associated with the vortices then has the form of such a wavepacket along the mixing layer. ${ }^{3,4}$ Such radiation by amplified hydrodynamic fluctuations also occurs at the trailing edge of a sharp-edged flat plate. ${ }^{5}$ A solution of this problem is critical to the efficient design of control strategies, because the latter may be different whether the acoustic power output is due to a high amount of energy brought by the hydrodynamic flow or a high efficiency through the conversion process into acoustics.

The acoustic response to generic pressure distributions is driven by a Cauchy problem whose direct numerical resolution as a partial differential equation is difficult. ${ }^{6,7}$ Two other techniques are available. The first one consists in the

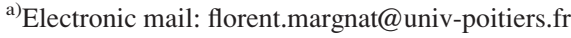

definition of a source term as the acoustic excitation; that is solving an inhomogeneous wave equation. The Lighthill formalism is the natural candidate to this aim. It has been carried out by $\mathrm{Obrist}^{8}$ who modeled the first component of the Lighthill tensor as a wavepacket, and investigated the role of its phase velocity and its spatial distribution in a multidimensional space, then extending to that of the group velocity. ${ }^{9}$ That author emphasized the predominant effects of those characteristics on directivity patterns. The same formalism has been used through analytical and experimental work by Papamoschou ${ }^{10}$ and Cavalieri et al. ${ }^{11}$ For jittering wavepackets in a middle subsonic flow, the temporal fluctuations of the envelope highlighted efficient conditions for sound radiation. The second technique is to excite the wave operator through the boundary conditions of the homogeneous wave equation as used by Avital and Sandham ${ }^{12}$ (see also Refs. 13-15, for instance). In particular cases, an analytical solution might be provided, as Crighton and Huerre ${ }^{16}$ did to suggest a theoretical explanation for the directivity of forced jets that Laufer and Yen ${ }^{17}$ observed in their experiments. The same track was followed by Fleury et al. ${ }^{18}$ on a cylindrical Kirchhoff surface in the context of round jet noise.

The acoustic energy radiated by a pressure distribution is triggered by its modes in the wavenumber spectrum lower than the acoustic wavenumber (supersonic phase speed $^{9,15,19,20}$ ). The localized spatial envelope yields an amplitude modulation in the wavenumber spectrum, instead of a single pic at the hydrodynamic wavenumber in the case of a wave without space modulation. Consequently, even for subsonic phase speed, there is energy in the supersonic range of the wavenumber spectrum. This is usually referred to as the supersonic tail of the wavepacket and can 
be considered as the purely radiating component of it. The remaining part of the wavenumber spectrum of the pressure distribution has mathematically no contribution to the acoustic field in the Kirchhoff formalism. It may be conceptually related to the silent base flow introduced by Sinayoko and Agarwal, ${ }^{21}$ whose physical existence and meaning has not yet been clearly established, however. On the other hand, which proportion does that acoustic energy represent with respect to the energy brought by the hydrodynamic wave needs further documentation, as well as the dependence of this proportion on the wavepackets characteristics.

The present paper follows the guideline set by Sandham et al. ${ }^{14}$ through numerical integration of the homogeneous Helmholtz equation in the Kirchhoff formalism, introducing the acoustic excitation as a boundary condition. Salient features of wavepackets are their shape, designed by the envelope, and the wavenumber of the traveling waves within the latter. The purpose of this study is to characterize the acoustic response of the medium to such generic excitation, through a parametric investigation on the envelope function, assumed static. The effect of both the hydrodynamic and the acoustic compactness of the wavepacket is investigated, varying the envelope length and the phase Mach number of the traveling wave, respectively. The effect of an asymmetrical envelope is specifically studied. The acoustic response is described by the acoustic efficiency of the wavepacket and how the acoustic intensity is distributed on the directivity range. Propagation in an acoustic medium at rest is first considered. Second, the analysis is extended when a uniform flow is present in the acoustic medium, using a convected Green function. It thus broadens possibilities toward flows where organized, unsteady motions may be identified as dominant aeroacoustic sources such as cavities ${ }^{22}$ and turbulent boundary layers. ${ }^{23}$

The paper is organized as follow: Section II explicits the theoretical background with the mathematical conventions hereby adopted for the resolution of the wave equation; the Kirchhoff formalism is introduced in a static, propagation medium and a formulation for a distant observer is given. Analytical developments yielded prediction for high efficiency conditions; the numerical tool is presented in Sec. III, and its range of validity and interest is exhibited by comparison with the results given by the analytical formulas. In Sec. IV, a selection of cases and results are provided to draw the effective conditions of a high rate of energy release to the acoustic medium. Finally, in Sec. V, the convected Green function is used to study the efficiency of wavepackets when a uniform flow is present in the acoustic medium. Additional practical consequences are viewed in Sec. VI.

\section{ANALYTICAL DEVELOPMENTS FOR A STATIC PROPAGATION MEDIUM}

\section{A. The Kirchhoff formalism}

We consider the wave equation for the acoustic pressure $\hat{p}(\mathbf{x}, t)$, in the superior half-plane for a bi-dimensional, static, uniform medium

$$
\frac{\partial^{2}}{\partial x_{1}^{2}} \hat{p}(\mathbf{x}, t)+\frac{\partial^{2}}{\partial x_{2}^{2}} \hat{p}(\mathbf{x}, t)-\frac{1}{c_{\infty}^{2}} \frac{\partial^{2}}{\partial t^{2}} \hat{p}(\mathbf{x}, t)=0
$$

with $x_{1} \in \mathbb{R}$ and $x_{2} \in \mathbb{R}^{+}$. The Fourier transform in time, defined as follows, is applied, yielding the homogeneous Helmholtz equation:

$$
\left\{\begin{array}{l}
f(\mathbf{x}, \omega)=\int_{-\infty}^{+\infty} \hat{f}(\mathbf{x}, t) e^{+i \omega t} \mathrm{~d} t \\
\hat{f}(\mathbf{x}, t)=\frac{1}{2 \pi} \int_{-\infty}^{+\infty} f(\mathbf{x}, \omega) e^{-i \omega t} \mathrm{~d} \omega
\end{array}\right.
$$

where $i$ is the imaginary unit and $\omega$ is the angular frequency. For one particular mode, one obtains

$$
\frac{\partial^{2}}{\partial x_{1}^{2}} p(\mathbf{x})+\frac{\partial^{2}}{\partial x_{2}^{2}} p(\mathbf{x})+k_{a}^{2} p(\mathbf{x})=0,
$$

with $k_{a}=\omega / c_{\infty}$. The excitation, assumed to be harmonic in frequency, is introduced through the boundary condition $\left(x_{2}\right.$ $=0$ ), in the following general form:

$$
p\left(x_{1}, 0\right)=P_{0} A\left(x_{1}\right) \exp \left(i \frac{\omega}{U_{c}} x_{1}\right),
$$

where $A$ is the envelope function, $U_{c}$ is the phase velocity of the convected wave, and $P_{0}$ is the pressure amplitude. The latter is taken as unity and will be omitted in the following, unless otherwise specified. The problem is sketched up in Fig. 1.

We resolve the Helmholtz Eq. (1) in the Kirchhoff formalism ${ }^{24}$ which yields the acoustic pressure according to the initial distribution

$$
p(\mathbf{x})=\int_{\Sigma(\mathbf{y})}\left\{G(\mathbf{x} \mid \mathbf{y}) \frac{\partial p(\mathbf{y})}{\partial \mathbf{n}}-p(\mathbf{y}) \frac{\partial G(\mathbf{x} \mid \mathbf{y})}{\partial \mathbf{n}}\right\} \mathrm{d} \Sigma(\mathbf{y}),
$$

$\Sigma(\mathbf{y})$ is the control surface and $\mathbf{n}$ its outward pointing normal. The two-dimensional (2D) free-space Green function associated with the above Fourier transform convention is

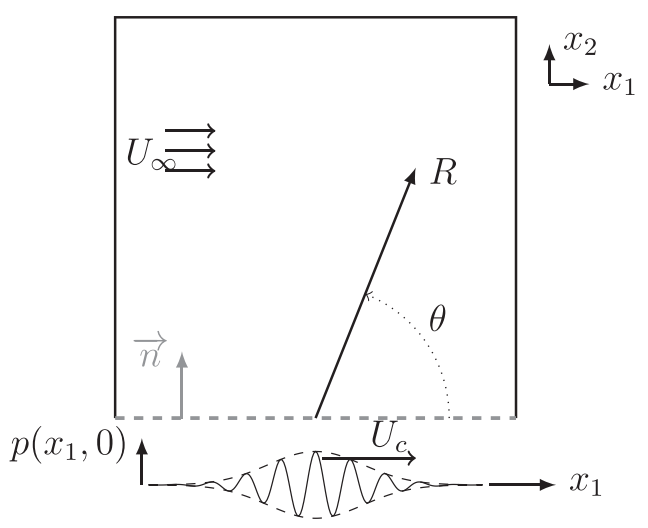

FIG. 1. Notations for the excitation of the wave equation by a pressure distribution at a boundary. $U_{\infty}=0$, except in Sec. V. 


$$
G(\mathbf{x} \mid \mathbf{y})=\frac{1}{4 i} H_{0}^{(1)}\left(k_{a} r\right)
$$

with $r=|\mathbf{x}-\mathbf{y}|$ and $H_{\nu}^{(1)}$ is the Hankel function of the first kind of order $\nu$. In Eq. (3), the pressure gradient normal to the control surface has to be prescribed in addition to the pressure itself. However, for a plane Kirchhoff surface and no other sources, the contributions of each of the two terms in Eq. (3) to the resulting integral are identical. ${ }^{25}$ For other (relatively simple) geometries, designing a tailored Green function can fix that issue out. ${ }^{26,27}$ Equation (3) then becomes

$$
p(\mathbf{x})=\int_{y_{2}=0}\left\{-2 p(\mathbf{y}) \frac{\partial G(\mathbf{x} \mid \mathbf{y})}{\partial y_{2}}\right\} \partial y_{1} .
$$

Deriving $G(\mathbf{x} \mid \mathbf{y})$ with respect to the coordinate $y_{2}$ yields

$$
\frac{\partial G(\mathbf{x} \mid \mathbf{y})}{\partial y_{2}}=\frac{1}{4 i} k_{a} \frac{r_{2}}{r} H_{1}^{(1)}\left(k_{a} r\right),
$$

where $r_{i}=x_{i}-y_{i}$. Formula (5) provides the acoustic pressure explicitly once the pressure distribution [Eq. (2)] is specified. It is valid in both the near- and far-fields, and, through a numerical integration, any distribution may be addressed. However, the far-field approximation is now introduced, allowing us to express the acoustic field as a function of the supersonic part of the pressure distribution in the longitudinal wavenumber space, and to derive analytical solutions for specific distributions. Regarding the asymptotic behavior of the Hankel function for large arguments

$$
H_{\nu}^{(1)}(z) \sim \sqrt{\frac{2}{\pi z}} e^{i(z-\nu(\pi / 2)-(\pi / 4))}
$$

and $r$ being approximated as $r \sim|\mathbf{x}|-\mathbf{x} \cdot \mathbf{y} /|\mathbf{x}|$ (for $|\mathbf{x}|$ $\gg|\mathbf{y}|$ ), the far-field expression of the Green function first derivative (for $y_{2}=0$ ) becomes

$$
\frac{\partial G(\mathbf{x} \mid \mathbf{y})}{\partial y_{2}} \sim \sin \theta \sqrt{\frac{k_{a}}{8 \pi R}} e^{i\left(k_{a} R-k_{a} y_{1} \cos \theta+(3 \pi / 4)\right)},
$$

with $R=|\mathbf{x}|, \sin \theta=x_{2} / R$, and $\cos \theta=x_{1} / R$. Substituting it inside Eq. (5) yields

$$
p(\mathbf{x}) \sim E \sin \theta \sqrt{\frac{k_{a}}{2 \pi R}} \int_{-\infty}^{+\infty} p(\mathbf{y}) e^{-i k_{a} \cos \theta y_{1}} \mathrm{~d} y_{1},
$$

where $E=\exp \left[i\left(k_{a} R-(\pi / 4)\right)\right]$. Introducing the Fourier transform of $p\left(y_{1}, 0\right)$ in the axial direction

$$
\left\{\begin{array}{l}
\tilde{f}(K)=\frac{1}{2 \pi} \int_{-\infty}^{+\infty} f\left(y_{1}\right) e^{-i K y_{1}} \mathrm{~d} y_{1} \\
f\left(y_{1}\right)=\int_{-\infty}^{+\infty} \tilde{f}(K) e^{+i K y_{1}} \mathrm{~d} K
\end{array}\right.
$$

gives the acoustic pressure as

$$
p(\mathbf{x}) \sim E \sin \theta \sqrt{\frac{2 \pi k_{a}}{R}} \tilde{p}\left(K=k_{a} \cos \theta\right) .
$$

Expression (9) shows that the acoustic response toward the direction $\theta$ in the far-field is proportional to the amplitude of the spatial mode $K=k_{a} \cos \theta$. Thus $|K| \leq k_{a}$, meaning that only those spatial modes of the pressure distribution with a wavenumber smaller than $k_{a}=M_{c} k_{h}$ are able to excite the acoustic far-field. They have thus been called the supersonic tail of the wavepacket, for their phase velocity $\omega / K$ is faster than the sound speed. Expression (9) also establishes a direct relation between the shape of the wavenumber spectrum in the range $\left[-k_{a} ;+k_{a}\right]$ and the directivity pattern, in agreement with previous studies using either the Kirchhoff formalism ${ }^{12,16}$ or a modeling of the Lighthill source term. ${ }^{8,28}$ The directivity pattern is weighted, however, by $\sin \theta$, exhibiting a dipolar behavior in the transverse direction, with sound cancellation in the axial direction. This feature comes from the normal derivative of the Green function (8) in the Kirchhoff formalism. Studies that use a source term to excite the wave equation, retaining only the longitudinal component of the Lighthill tensor, do not exhibit such a formal dependence on $\sin \theta$. Expression (9) thus returns the acoustic field once the wavenumber spectrum of the pressure distribution [Eq. (2)] is known, which can be brought by a numerical evaluation, as presented in Sec. III.

Before exploring a range of parameters for $A$ and $U_{c}$ in the pressure distribution [Eq. (2)], further analytical developments may be conducted for specific distributions leading to the explicit formula of the directivity and the acoustic efficiency. Crighton and Huerre ${ }^{16}$ addressed the case of envelopes $A$ with Gaussian, exponential, and algebraic decays along $y_{1}$, while only the Gaussian form received the most attention. ${ }^{8,11,13}$ Fleury et al. ${ }^{18}$ also proposed an envelope with two sinusoidal arches, representing the growth and decay of two instability modes in an excited jet.

A wavepacket model is relevant for two main reasons. First, experimental results for unforced jets ${ }^{11}$ showed that the axisymmetric mode of the acoustic field can be clearly associated with an axially non-compact source, in the form of a wavepacket. Now, the sound field for low polar angles (measured with respect to the jet axis) is found to be dominated by the axisymmetric mode, particularly at the peak Strouhal number. Second, Ffowcs Williams and Kempton ${ }^{2}$ modeled the development of the unforced jet by randomness in the phase velocity of the wavepacket, then predicted that the field shapes and spectra for the excited and unexcited jet are similar. Considering such a generality of the wavepacket mechanism in aerodynamic noise generation, its acoustic efficiency deserves further investigation, in addition to existing publications focused on directivity and restricted to jet noise. Only the Gaussian case with fixed phase velocity is addressed hereafter.

\section{B. Gaussian wavepacket}

A Gaussian, centered, static form is selected as the envelope function of the wavepacket defined as

$$
A\left(y_{1}\right)=\exp \left(-\frac{y_{1}^{2}}{\sigma^{2}}\right)
$$


where $\sigma$ is the parameter associated with the envelope length. The Fourier transform of the initial pressure distribution [Eq. (2)] then becomes

$$
\tilde{p}(K)=\frac{\sigma}{2 \sqrt{\pi}} e^{-\left(\sigma^{2} / 4\right)\left(k_{h}-K\right)^{2}} .
$$

Substituting $\tilde{p}(K)$ into Eq. (9) yields

$$
p(\mathbf{x}) \sim E \sigma \sin \theta \sqrt{\frac{k_{a}}{2 R}} e^{-\left(\sigma^{2} / 4\right)\left(k_{h}-k_{a} \cos \theta\right)^{2}} .
$$

For a low subsonic phase speed where $k_{a} \ll k_{h}$, this can be approximated by

$$
p(\mathbf{x}) \underset{M_{c} \rightarrow 0}{\sim} E \sigma \sin \theta \sqrt{\frac{k_{a}}{2 R}} e^{-\left(\sigma^{2} / 4\right) k_{h}^{2}} e^{\left(\sigma^{2} / 2\right) k_{h}^{2} M_{c} \cos \theta},
$$

with $M_{c}=k_{a} / k_{h}$ as the phase Mach number. This expression is the same as expression (3.7) in Crighton and Huerre ${ }^{16}$ who solve the Helmholtz equation using the stationary phase method. However, these authors assumed long envelopes with respect to the hydrodynamic wavelength, while such assumption is not used here. Further developments give access to the angle of maximum pressure radiation $\alpha$ defined by

$$
\left.\frac{\partial p(\mathbf{x})}{\partial \theta}\right|_{\theta=\alpha}=0
$$

whose two solutions are

$$
\alpha_{1,2}=\arccos \left[\frac{-1 \pm \sqrt{1+\left(\sigma^{2} k_{h}^{2} M_{c}\right)^{2}}}{\sigma^{2} k_{h}^{2} M_{c}}\right] .
$$

The positive one is plotted in Fig. 2(b). $\alpha$ decreases from $\pi / 2$ (the limit for $M_{c}=0$ ) as the quantity $\sigma^{2} k_{h}^{2} M_{c}$ increases. The emission is thus directed toward lower angles as the wavepacket is longer with respect to the hydrodynamic wavelength, or the phase speed is higher. Note that $\sigma^{2} k_{h}^{2} M_{c}=\sigma k_{h} \times \sigma k_{a}$, meaning that the hydrodynamic compactness and the acoustic compactness of the envelope multiply their influence on the angle of maximum pressure radiation.

\section{Acoustic efficiency}

Now the rate of energy released to the medium by the excitation is quantified through the acoustic efficiency $\eta$, defined as the ratio between the power $W_{h}$ provided as input by the pressure distribution and the power $W_{a}$ radiated as output

$$
\eta=W_{a} / W_{h},
$$

with

$$
W_{h}=\int_{y_{2}=0} \frac{\left|p\left(y_{1}, 0\right)\right|^{2}}{\rho_{0} c_{\infty} M_{c}} \mathrm{~d} y_{1} ; \quad W_{a}=R \int_{0}^{\pi} \frac{|p(R, \theta)|^{2}}{\rho_{0} c_{\infty}} \mathrm{d} \theta,
$$

where $|\phi|$ is the modulus of $\phi$. Using Eq. (9), the acoustic power is given by

$$
W_{a} \sim \frac{2 \pi k_{a}}{\rho_{0} c_{\infty}} \int_{0}^{\pi} \tilde{p}(K)^{2} \sin ^{2} \theta \mathrm{d} \theta
$$

The power expresses dependency upon the supersonic wavenumbers radiating at angle $\theta$ through the parameter $K=k_{a} \cos \theta$. For a simple Gaussian wavepacket, in the case of low subsonic flow, substitution of expression (11) yields

$$
W_{a} \underset{M_{c} \rightarrow 0}{\sim} \frac{\pi}{2 \rho_{0} c_{\infty} k_{h}} e^{-\left(\sigma^{2} k_{h}^{2} / 2\right)} I_{1}\left(\sigma^{2} k_{h}^{2} M_{c}\right),
$$

where $I_{n}(z)$ is the modified Bessel function of the first kind of integer order $n$. Note that there is no dependency on the radius $R$ as a consequence of the far-field approximation. Expliciting the integral over $y_{1}$ in the case of a Gaussian, centered wavepacket returns $W_{h}$ as

$$
W_{h}=\frac{\sigma}{\rho_{0} c_{\infty} M_{c}} \sqrt{\frac{\pi}{2}}
$$

exhibiting proportionality between the hydrodynamic power and the length of the Gaussian envelope. Combining those results yields the following analytical expression for the acoustic efficiency $\eta$, namely,

$$
\eta \underset{M_{c} \rightarrow 0}{\sim} \sqrt{\frac{\pi}{2} \frac{M_{c}}{k_{h} \sigma}} e^{-\left(\sigma^{2} k_{h}^{2} / 2\right)} I_{1}\left(\sigma^{2} k_{h}^{2} M_{c}\right) .
$$

Exhibiting $\sigma k_{h}$, expression (17) first shows that the hydrodynamic wavelength is the appropriate reference for the envelope extent $\sigma$ regarding the acoustic efficiency. In other words, hydrodynamic compactness is the driving parameter. Second, expression (17) shows that shorter wavepackets are more efficient, as explained by Obrist: ${ }^{8}$ the enlargement of the wavepacket envelope leads to a thinning of the spectral envelope around the hydrodynamic wavenumber. Thus, the radiating part of the excitation, localized in the range $\left[-k_{a} ;+k_{a}\right]$, will be lower, affecting in the same way the acoustic efficiency. Isocontours of $\eta$ given by Eq. (17) are plotted in Fig. 2(a), and exhibit a very large range of values, distributed on more than 20 orders of magnitude, from about $10^{-25}$ at low $M_{c}$ for long envelopes, to about $10^{-2}$ at relatively high $M_{c}$ for short envelopes. Differentiating Eq. (17), one can show that the acoustic efficiency reaches a maximum for a value of $\sigma$, noted $\sigma_{\max }$, by the solution of

$$
\sigma_{\max } k_{h}-\sqrt{\frac{3 I_{1}\left(\sigma_{\max }^{2} k_{h}^{2} M_{c}\right)}{2 M_{c} I_{0}\left(\sigma_{\max }^{2} k_{h}^{2} M_{c}\right)-I_{1}\left(\sigma_{\max }^{2} k_{h}^{2} M_{c}\right)}}=0 .
$$

That value is close to unity as is visible in Fig. 2(a) up to $M_{c}=0.5$. The amplitude of the highest efficiency point is driven by the phase Mach number $M_{c}$ though it is obtained for $\sigma k_{h} \approx 1$ whatever the phase Mach number $M_{c}$. More precisely, we observe that the value of the maximum acoustic 

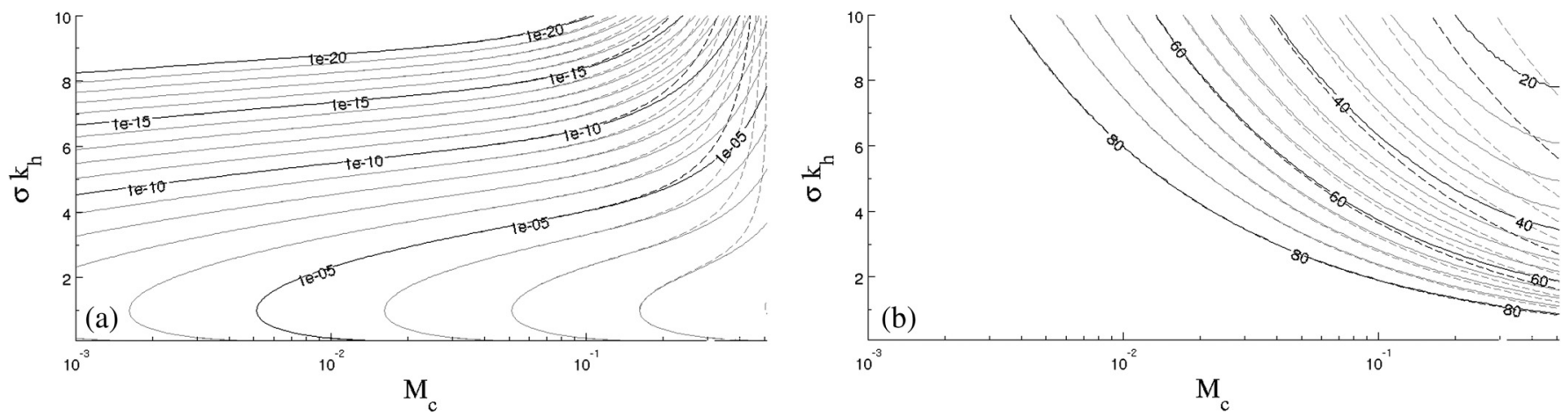

FIG. 2. Isocontours of efficiency (left) and of angle of maximum pressure radiation in degrees (right) for a Gaussian, centered, symmetric wavepacket. Comparison between analytical (dashed lines) and numerical (solid lines) results for the influence of the phase velocity and envelope length.

efficiency scales as $M_{c}^{2}$, while the value of $\sigma k_{h}$ for which the maximum is reached deviates from the unity with $M_{c}^{2}$ too. ${ }^{30}$

The analytical results of this section were obtained assuming low $M_{c}$. Their validity is thus questionable as $M_{c}$ is increasing, that is, as acoustic compacity of the hydrodynamic wave is lost. For high $M_{c}$, the integration giving the acoustic power has to be evaluated numerically, using known or computed wavenumber spectra of the pressure distribution. The numerical apparatus that computes either Eqs. (5) or (9) then Eq. (15) is presented in Sec. III.

\section{IMPLEMENTING THE KIRCHHOFF INTEGRAL}

\section{A. Tool presentation}

A numerical tool is developed to solve Eq. (1) in the Kirchhoff formalism in the far-field; that is, using Eq. (9). The length $L_{y_{1}}$ of the pressure distribution line is as long as 80 hydrodynamic wavelengths $\lambda_{h}=2 \pi / k_{h}$ with a numerical step $\delta y_{1}=\lambda_{h} / 40$ obtained from prior tests. ${ }^{29}$ The acoustic pressure is computed at five points for each degree on an observer circle at a given $R$. Both the Kirchhoff surface and the observer domain are centered in the axial direction (namely, $y_{1}$ and $x_{1}$, respectively). If the wavenumber spectrum of the pressure distribution is not known analytically, e.g., for asymmetric wavepackets, the specific mode $K=k_{a} \cos \theta$ for a regular set of angles $\theta$ is computed directly from the definition of the Fourier transform with trapezoidal rule integration. This reduces the computational cost with respect to standard fast Fourier transform routines, which would require an extremely long spatial window to obtain the desired resolution at the lowest wavenumbers. That procedure contains two sources of errors: on one hand, the rapid decay to the machine zero of the Gaussian envelope and its wavenumber spectrum, and on the other hand the windowing of the spatial envelope when evaluating the integral of the Fourier transform with finite bounds. In practice, at low phase velocities, a bounding of $\sigma$ was found around $12 / k_{h}$, after which the numerical procedure is affected by the round off error in the acoustic power integral and/or the secondary lobes due to the truncation of the envelope. Such bounding increases with the phase Mach number, and can be slightly mitigated by the use of quadruple precision or smarter integration algorithms.
In Fig. 2, isocontours of the acoustic efficiency of the wavepacket and of the angle of maximum emission are plotted as generated by the numerical tool described in this section compared to those given by the analytical expressions (17) and (14), respectively. For low phase velocities and long wavepackets, the agreement is perfect. When the phase velocity is increased, the analytical result loses validity and deviates from the numerical solution; this effect seeming more important for larger $\sigma$.

Further validation of the numerical tool is provided through the comparison of the pressure field, as given by the far-field approximation (9), the exact Kirchhoff integral (5), and the analytical expression (12) for a Gaussian, centered wavepacket. The acoustic response $|p(R, \theta)|^{2}$ depicted in Fig. 3 is observed at radii $R=\lambda_{a}$ and $R=5 \lambda_{a}$, for $\sigma=\lambda_{h}$ under a phase Mach number $M_{c}=k_{a} / k_{h}=0.05$. Perfect agreement is found at $R=5 \lambda_{a}$ while a slight discrepancy occurs for radius $R=\lambda_{a}$ between the exact Kirchhoff integral in Eq. (5) on one hand and the far-field approximation in Eq. (9) and the analytical expression (12) on the other hand. At a larger radius, comparing Eqs. (12) and (9) shows the good implementation of the Fourier transform with trapezoidal rule integration. Comparing Eq. (9) with expression (5) indicates that the far-field is reached soon, for the formulations match even at a radius $R=5 \lambda_{a}$. In the following, the results are computed from the far-field approximation of the Kirchhoff integral (9) for computation cost concerns.

\section{B. Parametric study}

The numerical tool is used to supplement the analytical study with results for configurations beyond its validity field, starting with a static acoustic medium and then extending the investigations to the propagation in a moving medium. Two cases are addressed, as presented in the following:

(1) The influence of higher phase velocities is first tested, from 0.002 to 0.900 , for the Gaussian, symmetric wavepacket. For $M_{c}=k_{a} / k_{h}$, the phase Mach number drives the acoustic compactness of both the traveling hydrodynamic wave and its envelope. To clarify the reading, the compactness of the traveling hydrodynamic wave will be identified as the wavelength compactness and the compactness of the envelope as envelope compactness, as 


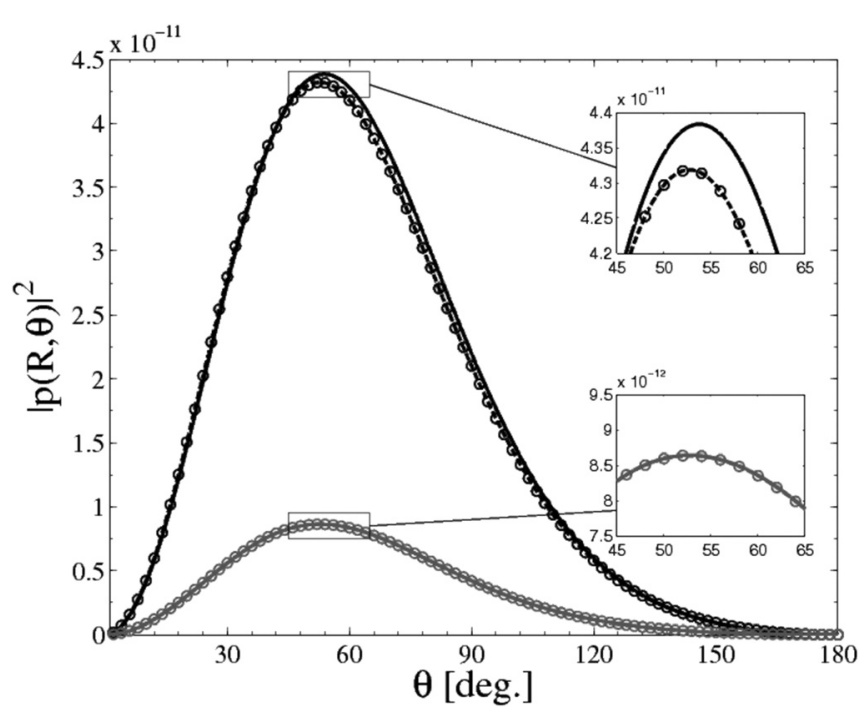

FIG. 3. Validation of the far-field approximation of the Kirchhoff integral (9) (dashed line) with the exact Kirchhoff integral (5) (solid line) and analytical expression (12) (symbols). $M_{c}=0.05 ; \sigma k_{h}=2 \pi$. Observed at $R=\lambda_{a}$ (black plots) and $R=5 \lambda_{a}$ (gray plots).

defined by the parameter $\sigma k_{a}$. The influence of $M_{c}$ on compactness is visible in the schematic of Fig. 4(a) where the longitudinal coordinate is scaled with the acoustic wavelength. The phase Mach number also pushes back the limitation in $\sigma$, so that longer envelopes can be studied [see Fig. 4(b)].

(2) Asymmetric cases are then tested, thus moving closer to practical hydrodynamic structures whose growth would be different from their decay. The wavepacket length is fixed in the upstream direction and is labeled $\sigma_{u}$ while $\sigma$ extends as for the symmetric case [Fig. 4(c)]. Such form of wavepacket can also be found in Refs. 10 and 20. It is worth noting that no difference is found regarding the orientation of the asymmetry (upstream or downstream $)^{29}$ whether in a static propagation medium or in a medium in motion.

The effects of these changes in the wavepacket envelope are quantified with the acoustic efficiency $\eta$ and angle of maximum emission $\alpha$ at a fixed radius $R$ in the far-field, as discussed in Sec. II. A wavepacket whose envelope is constituted by two halves of a Gaussian linked by a plateau is considered in Serré. ${ }^{30}$

\section{PROPAGATION IN A STATIC MEDIUM}

\section{A. Influence of the phase Mach number and the length of a Gaussian, centered, symmetric wavepacket}

The effects of the phase Mach number $M_{c}$ and the length $\sigma k_{h}$ are depicted in Fig. 5, both on the efficiency $\eta$ and the angle of emission $\alpha$. Increasing the phase velocity has two effects. The first one is a rise of the efficiency, expected from the loss of wavelength compactness, with an exponential shape. The second effect is an evolution of the directivity angle: for $M_{c}<0.4$, it is moved downward, then it reaches a plateau for intermediates $M_{c}$, before it goes upward again for $M_{c}>0.6$. Note also that for short wavepackets, the plateau is reached for lower values of $M_{c}$ and lasts longer. The behavior at low $M_{c}$ is consistent with observations of Moser et al. ${ }^{3}$ who reported such an effect of the convective Mach number on the noise directivity for subsonic mixing-layers. At a given phase velocity, the maximum of efficiency is reached around $\sigma k_{h}=1$, which does not deviate from the conclusions of the analytical study. The length of the wavepacket has a strong influence on the efficiency and directivity: short wavepackets have a strong efficiency and maximum radiation close to the transverse direction, while long wavepackets radiate toward low polar angles with low efficiency. That lowering of the directivity angle is faster when the phase velocity is increased.

\section{B. Influence of the phase Mach number and the length of a Gaussian, centered, asymmetric wavepacket}

When the growth of the wavepacket is different from the decay, the effects of the phase Mach number $M_{c}$ and the length $\sigma k_{h}$ are observed for two upstream lengths, namely, $\sigma_{u} k_{h}=2 \pi$ and $\sigma_{u} k_{h}=20 \pi$. These lengths are chosen for their qualitatively different behavior. The smallest length $\sigma_{u} k_{h}=2 \pi$ corresponds to an acoustically compact wavepacket, with high efficiency in the symmetrical case. The largest one is extended with a pronounced directivity in the symmetrical case. The behavior of the asymmetrical wavepacket shown in Figs. 6 and 7 exhibits singularities comparative to the symmetrical case. The falls of efficiency in Fig. 6(a) appears here when $\sigma=\sigma_{u}$, which is the symmetrical case. That is a noteworthy result, when the symmetrical case is long enough to be silent, its non-symmetric counterpart still emits noise. When $\sigma<\sigma_{u}$, the efficiency is increased by an increase of both $\sigma$ and $M_{c}$.
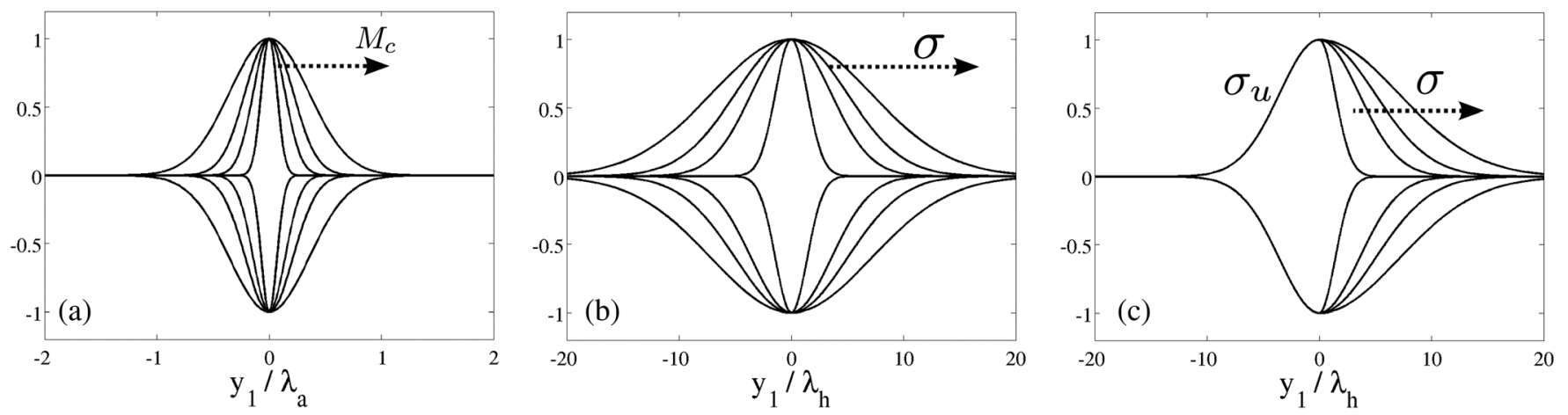

FIG. 4. Wavepacket shapes used in the parametric study. 

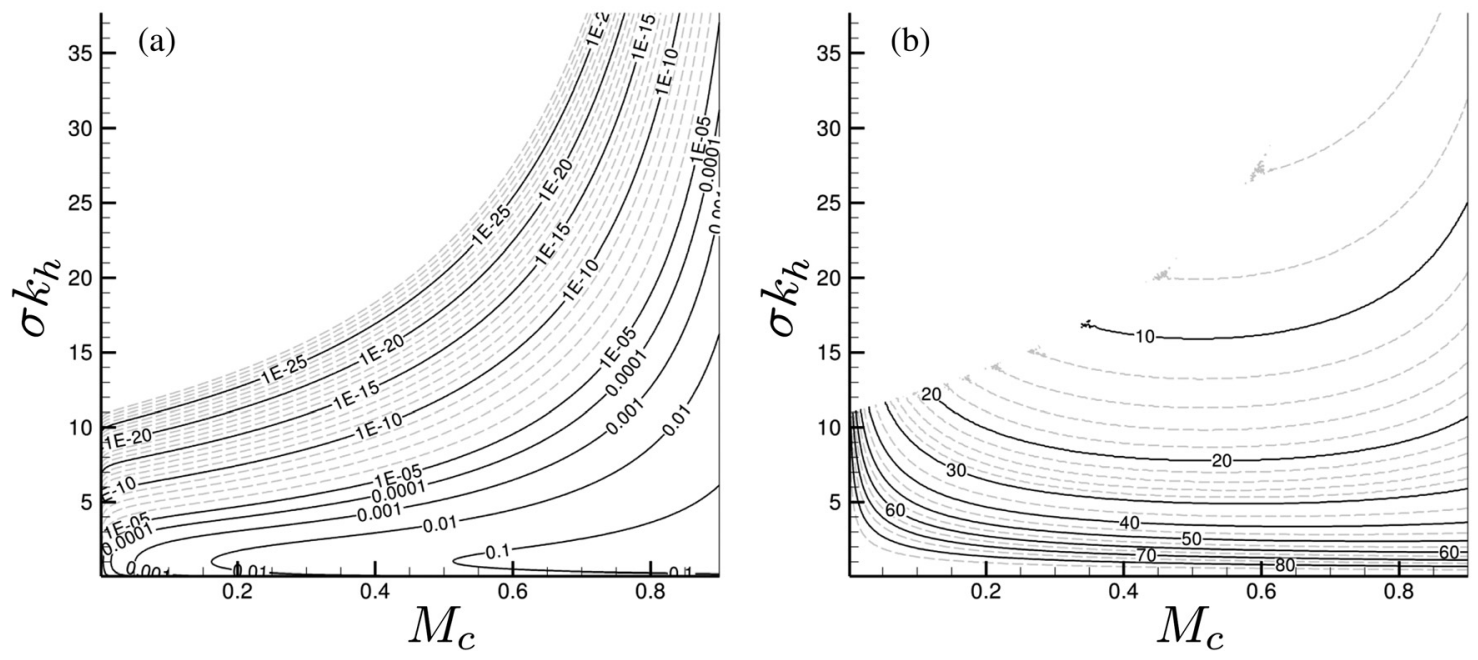

FIG. 5. Phase velocity and wavepacket length effects. Left: Isocontours of efficiency. Right: Isocontours of angle of maximum pressure radiation in degrees. Gaussian, centered, symmetric wavepacket.

When $\sigma>\sigma_{u}$, the efficiency is determined by the sole $M_{c}$. Moreover, due to the asymmetry, the angle of maximum emission for a given phase speed is almost only determined by the phase Mach number, irrespective of the length of the wavepacket as long as $\sigma$ is higher than a function like $\lambda_{h} /\left(1-M_{c}\right)$ [Figs. 6(b) and 7(b)]. Below, the emission angle is driven toward low polar angles both by the length and the phase Mach number. Such strong deviation from the behavior observed in the symmetric case [Fig. 5(b)] is worth noticing.

Those results lead to the conclusion that for a low Mach number, even a small loss of symmetry will lead to a strong increase of the acoustic efficiency. The directivity however will be more stable if the length is longer than the hydrodynamic wavelength.

\section{PROPAGATION IN A MOVING MEDIUM}

Considering a medium in motion will tell how the conclusions in a static medium remains suitable regarding

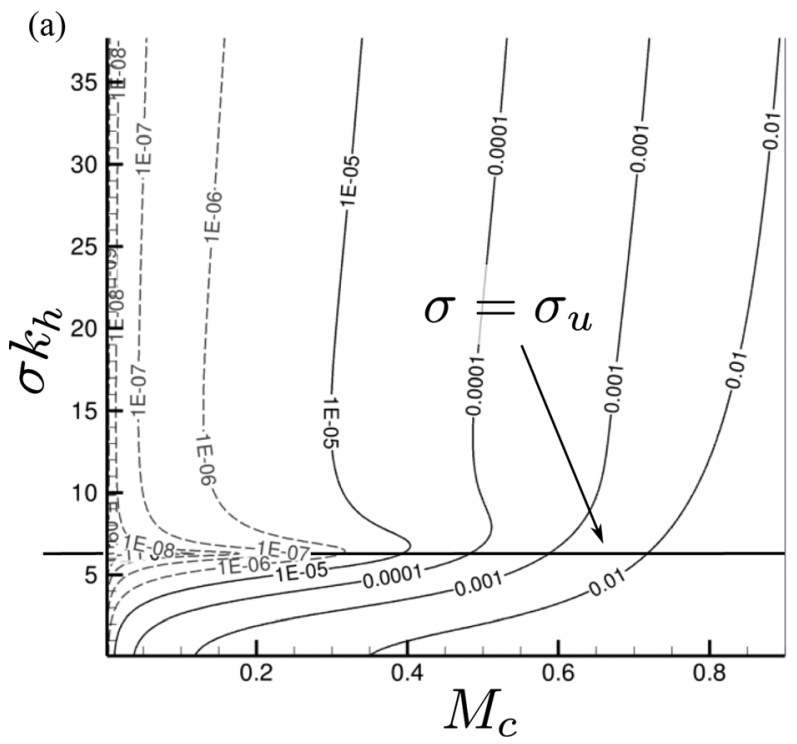

convected acoustic waves. It allows us to get closer from realistic flows where organized structures play a major role, such as co-flow around cruising jet, cavity, and turbulent boundary layer flows, for instance, where the sound waves are convected by the flow at infinity.

\section{A. Expression of the acoustic pressure}

To account for the presence of a uniform flow at $U_{\infty}=M_{\infty} c_{\infty}$ in the same direction as the hydrodynamic wave (see Fig. 1), the convected Green function is used. For 2D problems, it is given in the frequency domain by ${ }^{24}$

$$
G_{c}(\mathbf{x} \mid \mathbf{y})=\frac{1}{4 i \beta} \exp \left(i \frac{M_{\infty} k_{a} r_{1}}{\beta^{2}}\right) H_{0}^{(1)}\left(\frac{k_{a} r_{\beta}}{\beta^{2}}\right)
$$

with $\quad r_{i}=x_{i}-y_{i}, \quad \beta^{2}=1-M_{\infty}^{2}, \quad$ and $\quad r_{\beta}=\sqrt{r_{1}^{2}+\beta^{2} r_{2}^{2}}$. Deriving $G_{c}(\mathbf{x} \mid \mathbf{y})$ yields

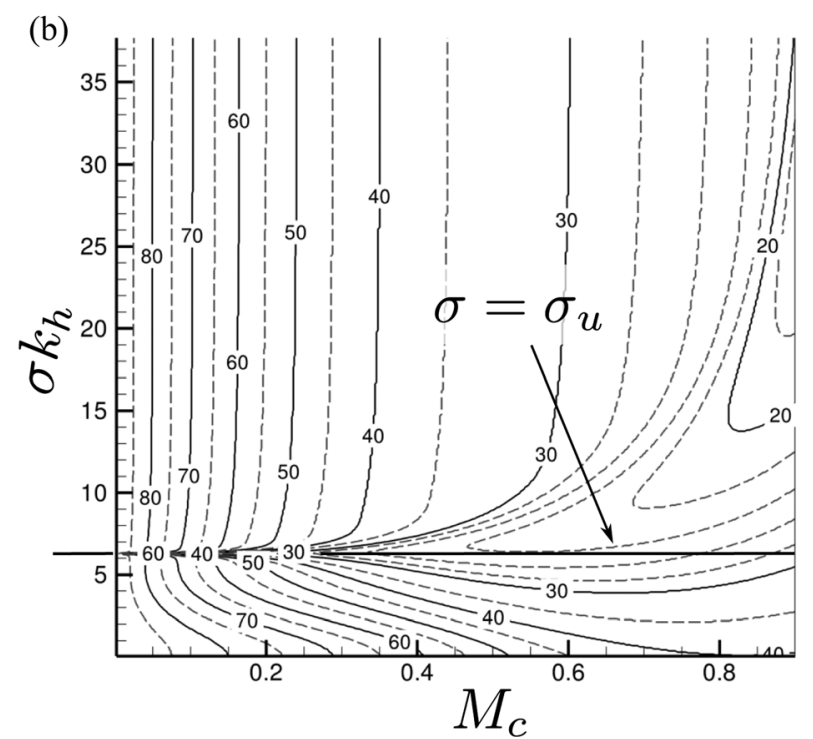

FIG. 6. Wavepacket length effects in the asymmetric case. Left: Isocontours of efficiency. Right: Isocontours of angle of maximum pressure radiation in degrees. $\sigma_{u} k_{h}=2 \pi$. 


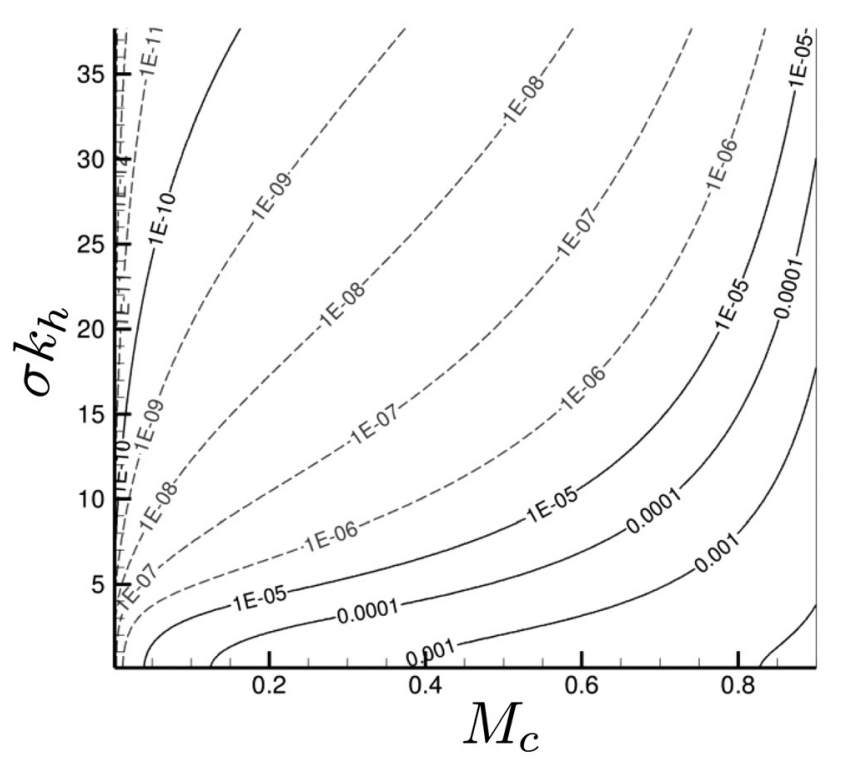

(a)

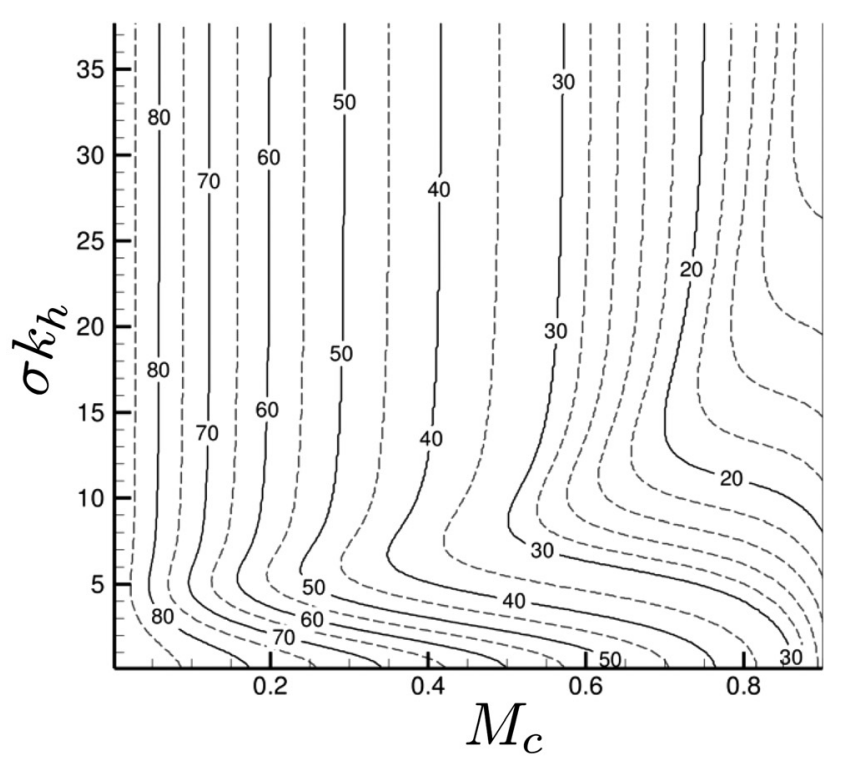

(b)

FIG. 7. Wavepacket length effects in the asymmetric case. Left: Isocontours of efficiency. Right: Isocontours of angle of maximum pressure radiation in degrees. $\sigma_{u} k_{h}=20 \pi$.

$$
\begin{aligned}
\frac{\partial G_{c}(\mathbf{x} \mid \mathbf{y})}{\partial y_{2}}= & \frac{1}{4 i \beta} k_{a} \frac{r_{2}}{r_{\beta}} \exp \left(i \frac{M_{\infty} k_{a} r_{1}}{\beta^{2}}\right) \\
& \times H_{1}^{(1)}\left(\frac{k_{a} r_{\beta}}{\beta^{2}}\right) .
\end{aligned}
$$

In the far-field one can write $r_{\beta} \underset{|\mathbf{x}| \gg|\mathbf{y}|}{\sim} x_{\beta}-\left(x_{1} / x_{\beta}\right) y_{1}$ with $x_{\beta}=\sqrt{x_{1}^{2}+\beta^{2} x_{2}^{2}}$. Using Eq. (7), Eq. (20) becomes

$$
\begin{aligned}
\frac{\partial G_{c}(\mathbf{x} \mid \mathbf{y})}{\partial y_{2}}= & \frac{1}{i} \sin \theta_{\beta} \sqrt{\frac{k_{a}}{8 \pi x_{\beta}}} \\
& \times e^{\left[i\left(k_{a} / \beta^{2}\right)\left(M_{\infty} x_{1}+x_{\beta}-\left(M_{\infty}+\left(x_{1} / x_{\beta}\right)\right) y_{1}\right)-3(\pi / 2)\right]},
\end{aligned}
$$

with $\sin \theta_{\beta}=x_{2} / x_{\beta}$. The Kirchhoff surface being aligned with the flow in the observer region, Eq. (5) still holds, and introducing the convected Green function derivative into it yields

$$
p(\mathbf{x}) \sim \sin \theta_{\beta} \sqrt{\frac{2 \pi k_{a}}{x_{\beta}}} \times e^{i\left[\left(k_{a} x_{\beta} / \beta^{2}\right)\left(M_{\infty} \cos \theta_{\beta}+1\right)-(\pi / 4)\right]} \hat{p}\left(K_{\beta}\right),
$$

for $K_{\beta}=k_{a}\left(M_{\infty}+\cos \theta_{\beta}\right) / \beta^{2}$ with $\cos \theta_{\beta}=x_{1} / x_{\beta}$. A new expression for the far-field approximation of the Kirchhoff integral is obtained. The acoustic response of an aeroacoustic excitation within a convected medium is then driven by the mode $K_{\beta}=k_{a}\left(M_{\infty}+\cos \theta_{\beta}\right) / \beta^{2}$ in the pseudo-direction $\theta_{\beta}$. Expliciting this expression in the case of a Gaussian, centered, symmetric wavepacket for a low phase Mach number yields

$$
\begin{aligned}
p(\mathbf{x}) \sim & \sigma e^{-\left(\sigma^{2} k_{h}^{2} / 4\right)} \sin \theta_{\beta} \sqrt{\frac{k_{a}}{2 x_{\beta}}} \\
& \times e^{i\left[\left(k_{a} x_{\beta} / \beta^{2}\right)\left(M_{\infty} \cos \theta_{\beta}+1\right)-(\pi / 4)\right]} \\
& \times e^{\left(\sigma^{2} k_{h}^{2} / 2\right)\left(M_{c} / \beta^{2}\right)\left(M_{\infty}+\cos \theta_{\beta}\right)} .
\end{aligned}
$$

Compared to expression (12), the convected acoustic factor modifies the space dependence through $x_{\beta}$ and $\theta_{\beta}$ instead of simply $r$ and $\theta$. Beyond that and the phase factor, the only effect brought by a mean flow in the observer domain is the factor $\exp \left(\sigma^{2} k_{h}^{2} / 2 \times M_{c} M_{\infty} / \beta^{2}\right)$ to the amplitude. It results in an increased pressure radiation as the flow velocity increases. Since the hydrodynamic power is unchanged, the acoustic efficiency may thus be enhanced by the same (squared) factor. Moreover, $M_{\infty}$ is added to $\cos \theta_{\beta}$, then influencing the directivity. The expression for the propagation in a medium at rest is recovered when $M_{\infty}$ is set to zero in Eq. (22).

The far-field approximation (22) is now computed using the tools developed for the static medium propagation case exposed earlier. The range of envelope lengths from Sec. IV is considered. The Mach number at infinity $M_{\infty}$ varies through conservation of a Mach number ratio defined as $M_{c}$ l $M_{\infty}$ since in real configurations, the external flow drives the convection of the coherent structures. For instance, is encountered a ratio $M_{c} / M_{\infty} \sim 0.3$ in boundary-layer flows, $M_{c} / M_{\infty} \sim 0.5$ in cavity flows, and eventually, $M_{c} / M_{\infty} \sim 0.7$ in wake flows. For every plotted situation, the maximum value for the Mach number at infinity ends up at $M_{\infty}=0.8$ then leading to different maximum values for $M_{c}$ for different Mach number ratios.

\section{B. Influence of the phase Mach number and the length of a Gaussian, centered, symmetric wavepacket}

Two lengths are considered here $\left(\sigma k_{h}=1\right.$ and $\left.\sigma k_{h}=10\right)$. The smallest length has been recognized as the length of maximum efficiency whatever the $M_{c}$ in Fig. 5(a). The range that covers the second length associated with the phase Mach number evolution enables to study cases for static propagation medium exhibiting directivity around $30^{\circ}$ for lower $M_{c}$, a plateau of directivity on very low polar angles and a new rise toward higher polar angles for higher $M_{c}$ [Fig. 5(b)]. Figure 8 exhibits those two lengths at the three 
Mach number ratios that were selected for their fair representation of real flow configurations. The corresponding static case $M_{\infty}=0$ is added for comparison. The phase Mach number is set on the horizontal axis and its maximum value is yielded from the Mach number ratio as explained above. Figure 8(a) displays the acoustic efficiency $\eta$, and Fig. 8(b) the angle of maximum emission.

The increase of the acoustic efficiency with $M_{c}$ and $M_{\infty}$ follows what was expected from the analytical result [Eq. (23)]. The previous conclusions regarding the phase Mach number $M_{c}$ effects on efficiency still hold for a moving medium, that is, at a given length, the higher $M_{c}$, the higher efficiency [Fig. 8(a)]. The length $\sigma$ has a major impact on the efficiency $\eta$. A smaller length leads to a lower efficiency as mentioned in the static case. The length $\sigma k_{h}=1$ is efficient and is not much influenced by the motion of the observer medium, the behavior being similar to the static case for lower $M_{c}$. The length $\sigma k_{h}=10$ is less efficient at lower $M_{c}$, but rapidly reaches high efficiency conditions, until meeting the shorter case $\left(\sigma k_{h}=1\right)$ and, consequently, is more dependent on the flow regime. Eventually, when $M_{\infty}=0.8$ (end of the curves), the same order of magnitude is reached for every length and every Mach ratio, reaching almost $60 \%$ in the noisiest situation. Up to a specific value of Mach numbers, the efficiency does not depend on the length any more. Such high efficiency is noteworthy, as far as aeroacoustics is concerned.

It has been seen that the rise of the phase Mach number drives the emission angle toward low polar angles before a plateau and a new rise. The same behavior is found when increasing the Mach number ratio, leading to the conclusion that the phase Mach number has significant effects. Two trends can be identified in Fig. 8(b). For low lengths, the emission angle seems to be weakly affected, exhibiting a steady value. For high lengths, the displacement of the emission angle toward low polar angles is emphasized as well as the plateau and the new rise around $M_{\infty}=0.5$, represented in Fig. 8(b) by the dashed, gray straight lines.

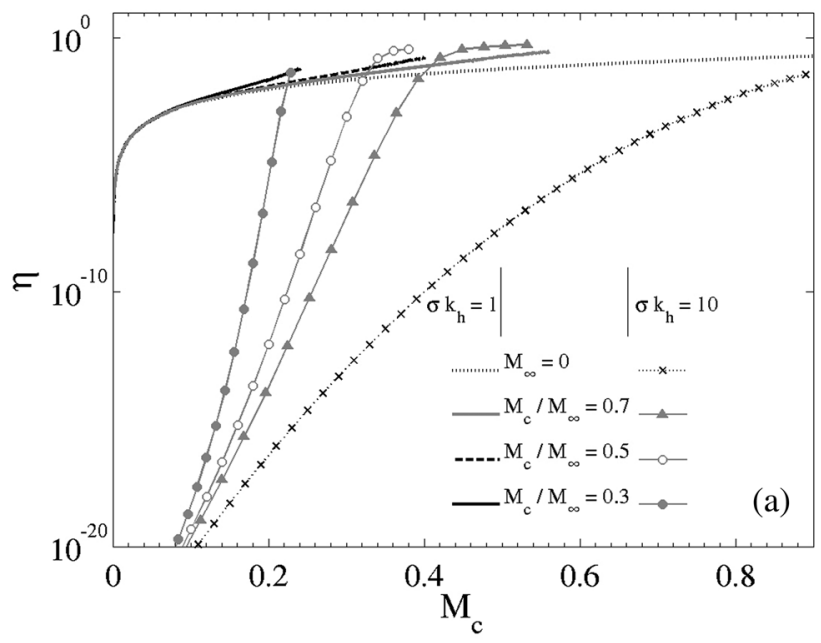

The truth is, the same behavior does occur for both plotted lengths, though it is reinforced for higher $\sigma$ : a specific value for the phase Mach number $M_{c}$ is selected according to its Mach number ratio, beyond which the dependency on $\sigma$ is eradicated concerning the efficiency and to a lesser extent, the angle of maximum emission. That selection provides high efficiency conditions and radiation toward the transverse direction. Eventually, the Mach number at infinity moves the directivity toward the transverse direction, while the case $M_{\infty}=0$ exhibits a continuous decay toward low polar angles, except a very shy rise for longer wavepackets.

\section{Influence of the length of an asymmetric wavepacket}

For convected acoustics, the influence of the length of an asymmetric wavepacket is depicted in Fig. 9 for the acoustic efficiency [Fig. 9(a)] and the angle of maximum emission [Fig. 9(b)]. The upstream lengths depicted below are set as the lengths investigated in the static case, namely, $\sigma_{u} k_{h}=2 \pi$ and $\sigma_{u} k_{h}=20 \pi$. Two downstream lengths are tested such as $\sigma k_{h}=\pi$ and $\sigma k_{h}=4 \pi$ to avoid both the symmetric case and the singular value when the wavepacket scales as the hydrodynamic wavelength as emphasized in the static case (see Figs. 6 and 7). The Mach number ratio $M_{c} l$ $M_{\infty}$ is set constant so as the Mach number at infinity is twice the phase Mach number in addition to the case $M_{\infty}=0$ for comparison. For all plotted lengths of wavepacket, the efficiency $\eta$ is increased by the convection velocity in the observer domain as soon as $M_{c} \geq 0.2$. For higher $\sigma_{u}$, the efficiency decreases and this trend is accentuated by a higher length $\sigma$ [Fig. 9(a)]. All the lengths do converge when $M_{\infty}=0.8$ as for the symmetric case at nearly $\eta=40 \%$. A point worth noting is the same trend in three steps in the emission angle as highlighted previously (see Figs. 5 and 8); although the plateau is skipped, the decrease toward low polar angle lasting longer and being stronger than the symmetric case, before the new rise when $M_{\infty}=0.5$ (or $M_{c}=0.25$ ).

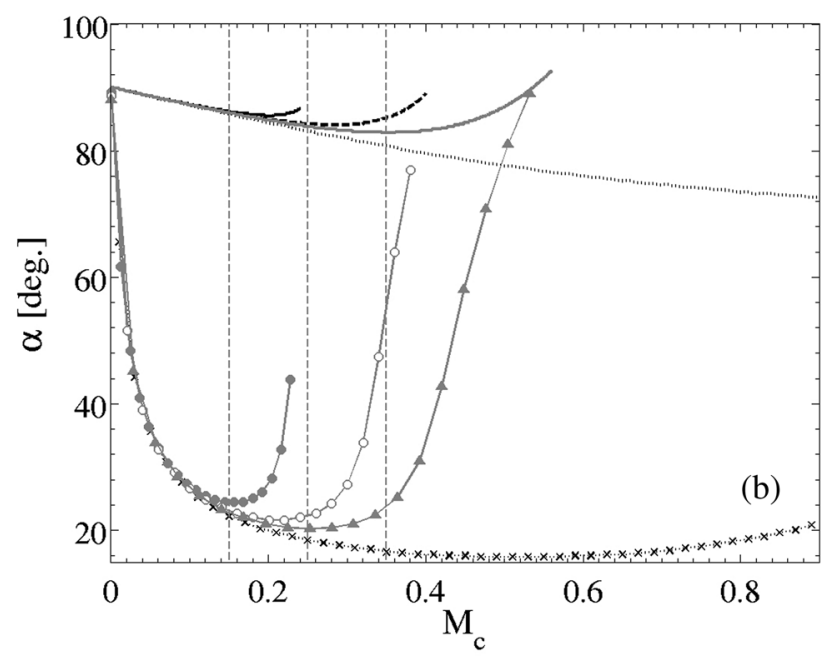

FIG. 8. Effects of convection in the observer medium on the acoustic emission of wavepackets. Symmetric case. Left: Efficiency. Right: Angle of maximum pressure radiation. The dashed, gray straight lines mark $M_{\infty}=0.5$ for the corresponding $M_{c}$ with respect to the Mach number ratio. 

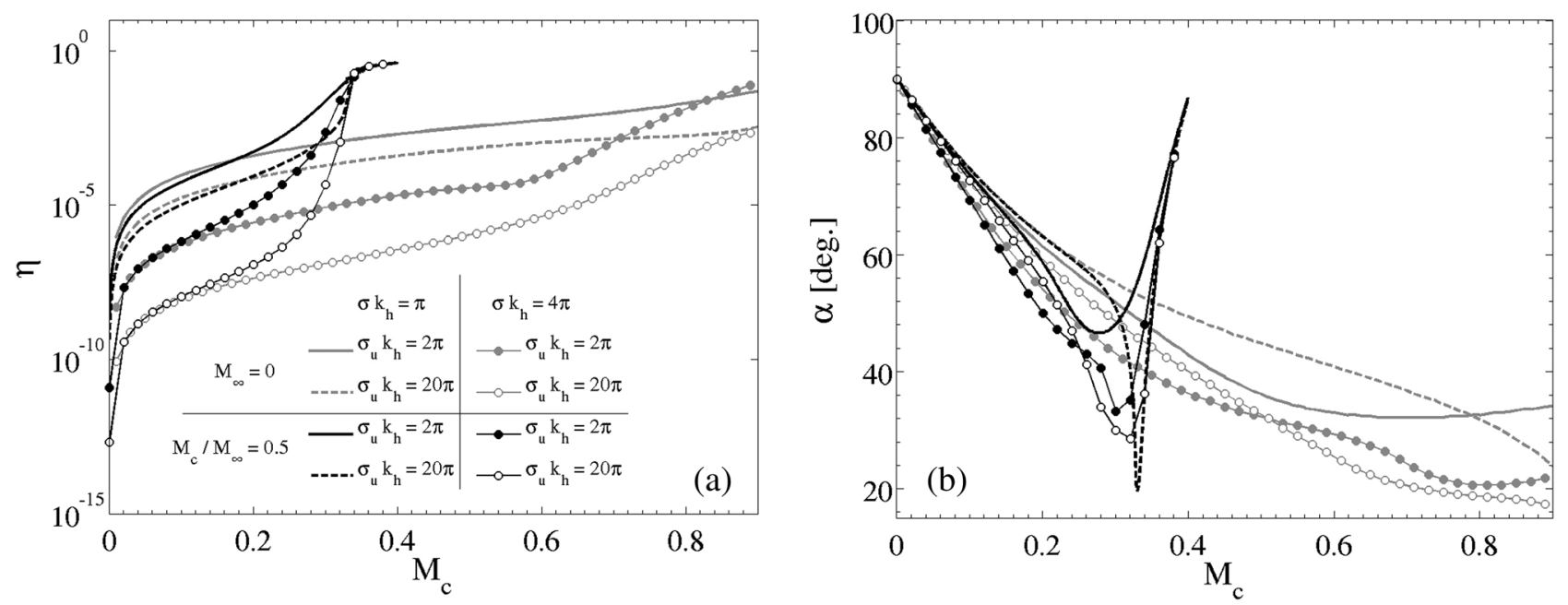

FIG. 9. Effects of convection in the observer medium on the acoustic emission of wavepackets. Asymmetric case. Left: Efficiency. Right: Angle of maximum pressure radiation.

The same conclusion as for the symmetric case is drawn, namely, a specific value for $M_{c}$ is selected and beyond that value, the dependency on $\sigma$ is lost, for both the efficiency and the angle of maximum emission converge whatever the length of the wavepacket. The minimum value for the emission angle, that is, the peak occurring at $M_{\infty}=0.5$ is reinforced as the length is increased [Fig. 9(b)]. This trend has been emphasized in the static case [Figs. 6(b) and 7(b)], namely, increasing the length will bring the directivity toward lower angles. Computations for higher Mach number ratio $M_{c} / M_{\infty}$ exhibit the very same trend, except for the singularity, namely, the rise of both efficiency and emission angle occurs for lower $M_{\infty}$.

\section{CONCLUDING REMARKS}

The homogeneous Helmholtz equation was resolved in the Kirchhoff formalism in order to characterize and investigate the acoustic response of the medium to a generic excitation. The excitation was applied to the wave operator through a pressure distribution at a boundary condition, with a generic Gaussian shape. The acoustic response was characterized both numerically and analytically by two criteria: (i) the angle of maximum emission; (ii) the rate of energy actually released to the far-field, namely, the acoustic efficiency. A better distinction is allowed between high efficiency conditions or high hydrodynamic power brought by the excitation. That second criterion has been less investigated before in the wavepacket approach. The study could have been conducted for a three-dimensional (3D) problem with a cylindrical control surface, but we did not want to restrict it to the jet configuration. A plane study, however, can represent radiation from, e.g., boundary layer or cavity flows, while then a 3D case would have brought more complexity in the parametric study.

A general conclusion is that acoustic efficiency spreads over several (about 20) orders of magnitude, depending on the envelope shape, on its length, and on the wavepacket phase Mach number. This wide range of values means that much could be expected from control strategies, in particular, at low phase Mach numbers. A numerical tool has been designed in order to address long envelopes, asymmetric envelopes, and high phase velocity of the hydrodynamic wave. We started with acoustic propagation in a medium at rest, then extended the problem to the presence of a uniform flow in the propagation region. For a Gaussian envelope, analytical developments and numerical computations emphasize a maximum efficiency when the wavepacket scales as the hydrodynamic wavenumber. The amplitude for this maximum efficiency scales as the second power of the phase velocity. On the other hand, the angle of maximum emission is predicted to be $\pi / 2$ without phase speed, then decreases by increasing either the length or the phase velocity of the wavepacket. Increasing the phase speed moves the emission angle downstream, before expressing a plateau and rising again. In the case of a Gaussian wavepacket with nonsymmetric features, it is more efficient and steadier on its maximum emission angle comparative to the corresponding symmetric case.

The use of the convected Green function allowed us to derive an original, analytical expression for the acoustic field accounting for the presence of a uniform flow in the propagation medium. It shows that the Mach number at infinity adds its influence exponentially with the phase velocity to the acoustic pressure radiation and the resulting efficiency. With asymmetric features, the emission angle is less likely to express a plateau, its directivity resisting to approach the low polar angles.

In order to give a clue for practical concerns from the present results, typical values are provided in Table I for a selection of envelopes and covering subsonic regime of the Mach numbers. The acoustic efficiency is reported, as well as the sound pressure level (SPL) estimated in the direction of maximum emission at 100 acoustic wavelengths, which corresponds to $34 \mathrm{~m}$ in air for a frequency of $1 \mathrm{kHz}$, for a wavepacket amplitude $P_{0}$ of $100 \mathrm{~Pa}$. For a propagation in a static medium, about $95 \mathrm{~dB}$ are obtained at $M_{c}=0.4$, for the 
TABLE I. Bounding values of the acoustic efficiency and SPL in the direction of maximum emission $(\theta=\alpha)$ at the distance $R=100 \lambda_{a}$, for $P_{0}=100$ Pa.

\begin{tabular}{|c|c|c|c|c|}
\hline & \multicolumn{2}{|c|}{$\begin{array}{c}\text { Static observer } \\
\text { medium } M_{c} \text { varies from } 0.05 \text { to } 0.4\end{array}$} & \multicolumn{2}{|c|}{$\begin{array}{c}\text { Observer medium in motion at } M_{\infty} \\
\text { varying from } 0.1 \text { to } 0.8 \text { with } M_{c}=0.5 M_{\infty}\end{array}$} \\
\hline & Acoustic efficiency $\eta$ & SPL $[\mathrm{dB}]$ & Acoustic efficiency $\eta$ & SPL $[\mathrm{dB}]$ \\
\hline Gaussian, centered, symmetric wavepacket $\sigma k_{h}=1$ & $9.5 \times 10^{-4} \rightarrow 6.1 \times 10^{-2}$ & $75 \rightarrow 93$ & $9.7 \times 10^{-4} \rightarrow 2.6 \times 10^{-1}$ & $75 \rightarrow 102$ \\
\hline Gaussian, centered, asymmetric wavepacket $\sigma k_{h}=8 \pi, \sigma_{u} k_{h}=2 \pi$ & $1.1 \times 10^{-7} \rightarrow 2.7 \times 10^{-5}$ & $47 \rightarrow 74$ & $1.2 \times 10^{-7} \rightarrow 4.0 \times 10^{-1}$ & $47 \rightarrow 126$ \\
\hline Gaussian, centered, asymmetric wavepacket $\sigma k_{h}=8 \pi, \sigma_{u} k_{h}=20 \pi$ & $8.3 \times 10^{-11} \rightarrow 1.3 \times 10^{-8}$ & $21 \rightarrow 44$ & $8.7 \times 10^{-11} \rightarrow 4.0 \times 10^{-1}$ & $21 \rightarrow 135$ \\
\hline
\end{tabular}

most efficient, symmetric wavepacket, and for the top-hat envelope. ${ }^{30}$ Note that in the former case, such SPL is due to a high efficiency, while it is due to high hydrodynamic power input in the latter case, with an efficiency thousand times smaller. Though the resulting SPL is the same in both cases, the sound production mechanisms are not, what may be of consequence as far as the design of control strategies is concerned. When the medium is set in motion, the values at the lowest Mach number are almost unchanged, but in the high subsonic regime, the effect of convected acoustics drastically enhances the noise. For instance, for $M_{\infty}=0.8$ and a Mach number ratio of 0.5 , the highest value of $M_{c}$ is the same as that in the static medium case, while the SPL is greater by 10 to $90 \mathrm{~dB}$. The noisiest configuration being then the asymmetric wavepacket with long envelope, which combines high efficiency and high hydrodynamic power input, with $135 \mathrm{~dB}$ in the direction of maximum emission.

The wavepacket approach for aerodynamic noise notwithstanding, the present results provide a guideline for the analysis of pressure distributions in the near-field when the acoustic response is targeted. Those obtained for propagation in a static medium can be applied in mixing layer or jet configuration. The extension of the approach to the convected Helmholtz equation allows using them in other flow configurations of interest. For instance, wakes, cavity configurations, or boundary layers may be considered. In such configurations, the Mach number at infinity is higher than the phase Mach number of the hydrodynamic wave, which is slowed down by a reverse flow or a no-slip condition at the wall.

\section{ACKNOWLEDGMENTS}

This research is granted from the French Ministry of Higher Education and Research. The authors thank Xavier Gloerfelt and express their gratitude to Professor Patrick Huerre for helpful discussions.

\section{NOMENCLATURE}

$\begin{aligned} A & \text { wavepacket's envelope function } \\ c_{\infty} & \text { sound velocity } \\ G & \text { Green's function } \\ G_{c} & \text { convected Green's function } \\ i & \text { imaginary unit } \\ k_{a}=\omega / c_{\infty} & \text { acoustic wavenumber } \\ k_{h}=\omega / U_{c} & \text { hydrodynamic wavenumber } \\ M_{\infty}=U_{\infty} / c_{\infty} & \text { convection Mach number } \\ M_{c}=U_{c} / c_{\infty} & \text { phase Mach number of the hydrodynamic } \\ & \text { wave }\end{aligned}$

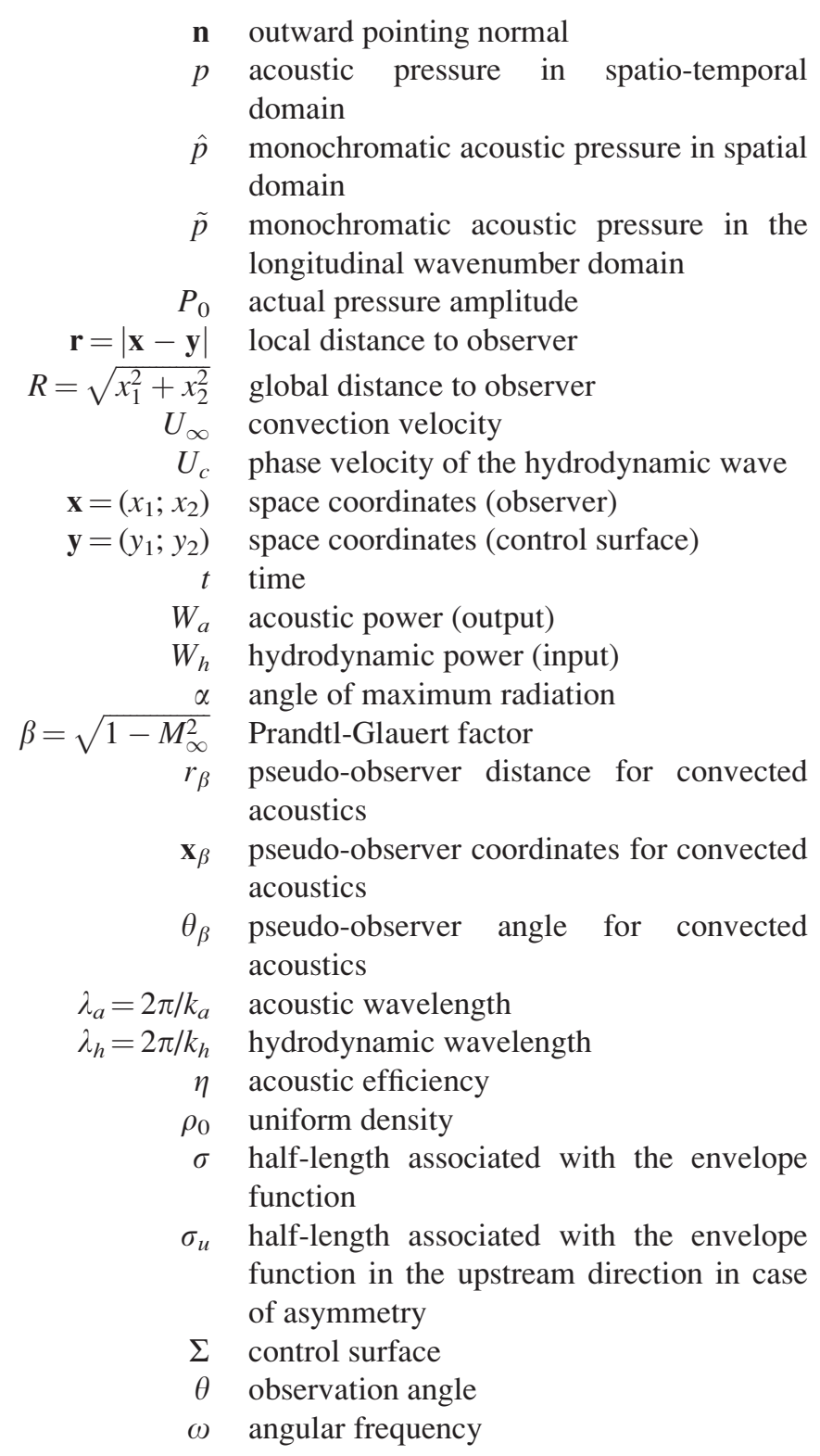

${ }^{1}$ P. Jordan and T. Colonius, "Wave packets and turbulent jet noise," Ann. Rev. Fluid Mech. 45, 173-195 (2013).

${ }^{2}$ J. E. Ffowcs Williams and A. J. Kempton, "The noise from the large-scale structure of a jet," J. Fluid Mech. 84, 673-694 (1978).

${ }^{3}$ C. Moser, E. Lamballais, F. Margnat, V. Fortuné, and Y. Gervais, "Numerical study of Mach number and thermal effects on sound radiation by a mixing layer," Int. J. Aeroacoust. 11, 555-580 (2012).

${ }^{4} \mathrm{~F}$. Margnat and X. Gloerfelt, "On compressibility assumptions in aeroacoustic integrals: A numerical study with subsonic mixing layers," J. Acoust. Soc. Am. 135, 3252-3263 (2014). 
${ }^{5}$ D. J. Moreau, L. A. Brooks, and C. J. Doolan, "On the aeroacoustic tonal noise generation mechanism of a sharp-edged plate," J. Acoust. Soc. Am. 129, EL154-EL160 (2011).

${ }^{6} \mathrm{~L}$. Marin, "An alternating iterative MFS algorithm for the Cauchy problem for the modified Helmholtz equation," Comput. Mech. 45, 665-677 (2010).

${ }^{7} \mathrm{C}$. Tajani, J. Abouchabaka, and O. Abdoun, "KMF algorithm for solving the Cauchy problem for Helmholtz equation," Appl. Math. Sci. 6, 4577-4587 (2012).

${ }^{8} \mathrm{D}$. Obrist, "Directivity of acoustic emissions from wave packets to the far field," J. Fluid Mech. 640, 165-186 (2009).

${ }^{9}$ D. Obrist, "Acoustic emissions from convected wave packets," Phys. Fluids 23, 026101 (2011)

${ }^{10}$ D. Papamoschou, "Prediction of jet noise shielding," in 48th AIAA Conference, AIAA2010-653 (2010).

${ }^{11}$ A. V. G. Cavalieri, P. Jordan, T. Colonius, and Y. Gervais, "Axisymmetric superdirectivity in subsonic jets," J. Fluid Mech. 704, 388-420 (2012).

${ }^{12}$ E. J. Avital and N. D. Sandham, "A note on the structure of the acoustic field emitted by a wave packet," J. Sound Vib. 204, 533-539 (1997).

${ }^{13}$ E. J. Avital, N. D. Sandham, and K. H. Luo, "Mach wave radiation by mixing layers. Part I: Analysis of the sound field," Theor. Comput. Fluid Dyn. 12, 73-90 (1998).

${ }^{14}$ N. D. Sandham, C. L. Morfey, and Z. W. Hu, "Sound radiation from exponentially growing and decaying surface waves," J. Sound Vib. 294, 355-361 (2006).

${ }^{15}$ E. J. Avital, R. E. Musafir, and T. Korakianitis, "Nonlinear propagation of sound emitted by high speed wave packets," J. Comput. Acoust. 21, 1-21 (2013).

${ }^{16}$ D. G. Crighton and P. Huerre, "Shear-layer pressure fluctuations and superdirective acoustic sources," J. Fluid Mech. 220, 355-368 (1990).

${ }^{17}$ J. Laufer and T. C. Yen, "Noise generation by a low Mach number jet," J. Fluid Mech. 134, 1-32 (1983).

${ }^{18}$ V. Fleury, C. Bailly, and D. Juvé, "Shear-layer acoustic radiation in an excited subsonic jet: Models for vortex pairing and superdirective noise," C. R. Mécanique 333, 754-761 (2005).
${ }^{19}$ A. P. Dowling and T. P. Hynes, "Sound generation by turbulence," European J. Mech. B/Fluids 23, 491-500 (2004).

${ }^{20}$ M. Koenig, A. V. G. Cavalieri, P. Jordan, J. Delville, Y. Gervais, and D. Papamoschou, "Far-field filtering and source imaging of subsonic jet noise," J. Sound Vib. 332, 4067-4088 (2013).

${ }^{21}$ S. Sinayoko and A. Agarwal, "The silent base flow and the sound sources in a laminar jet," J. Acoust. Soc. Am. 131, 1959-1968 (2012).

${ }^{22} \mathrm{X}$. Gloerfelt, "Compressible proper orthogonal decomposition/Galerkin reduced-order model of self-sustained oscillations in a cavity," Phys. Fluids 20, 115105 (2008).

${ }^{23} \mathrm{X}$. Gloerfelt and J. Berland, "Turbulent boundary-layer noise: Direct radiation at Mach number 0.5," J. Fluid Mech. 723, 318-351 (2013).

${ }^{24} \mathrm{X}$. Gloerfelt, C. Bailly, and D. Juvé, "Direct computation of the noise radiated by a subsonic cavity flow and application of integral methods," J. Sound Vib. 266, 119-146 (2003).

${ }^{25}$ A. P. Dowling and J. E. Ffowcs Williams, Sound and Sources of Sound (Ellis Horwood, Chichester, 1983).

${ }^{26} \mathrm{D}$. M. Chase, "Sound radiated by turbulent flow off a rigid half-plane as obtained from a wave vector spectrum of hydrodynamic pressure," J. Acoust. Soc. Am. 52, 1011-1023 (1972).

${ }^{27}$ O. Léon and J.-P. Brazier, "Investigation of the near and far pressure fields of dual-stream jets using an Euler-based PSE model," in 19th AIAA/CEAS Aeroacoustics Conference (2013).

${ }^{28}$ A. V. G. Cavalieri, G. Daviller, P. Comte, P. Jordan, G. Tadmor, and Y. Gervais, "Using Large Eddy Simulation to explore sound-source mechanisms in jets," J. Sound Vib. 330, 4098-4113 (2011).

${ }^{29}$ R. Serré and F. Margnat, "Modeling aeroacoustic excitations by subsonic wave packets in the Kirchhoff formalism," in 21e Congrès Français de Mécanique, Bordeaux, France (2013).

${ }^{30} \mathrm{R}$. Serré, "Contribution au développement d'une analogie vibroacoustique pour la modélisation du bruit d'origine aérodynamique" ("Contribution to the development of a vibro-acoustic analogy for modeling aerodynamic noise"), Ph.D. thesis, Arts et Métiers ParisTech (2014). 